Elke Gilson

\title{
"Zwiesprache mit Geistern": Die Entschränkung der Rhetorik im Werk von Monika Maron nach 1989
}

The texts published by the former East German author Monika Maron after the Wende clearly contribute to the intensified turn towards the past in German literature since 1989. Her texts from the pre-Wende era, however, deserve to be characterised as literature of remembrance as well. The changes brought on by the demise of the GDR manifest themselves not so much in the themes or structure of Maron's works - as was suggested elsewhere - as in the ways the voices of the past are dealt with before and after the disappearance of the East German state. The evolution at hand shows an increasing openness towards the legacy of (literary) predecessors. This article therefore proposes to focus on the intertextual memory of Monika Maron's oeuvre. Starting from the little-studied play Ada und Evald and the treatment it contains of the conceptualisation of literary communication by Heiner Müller, different stages of an evolution that might be termed a gradual return of rhetoric will be traced.

Die Nachwendeprosa der vormals ostdeutschen Autorin Monika Maron legt von der vielbeschworenen intensivierten Hinwendung zur Vergangenheit in der deutschen Literatur nach 1989 ein deutliches Zeugnis ab. Eine Charakterisierung als "Erinnerungsliteratur" erlauben aber auch schon ihre vor der Wende veröffentlichten Texte. Was sich mit dem Verschwinden der DDR verändert hat, ist weniger die Thematik oder der Aufbau ihrer Werke, als der Umgang mit den Stimmen der Vergangenheit. Die fragliche Evolution manifestiert sich deutlich in einer zunehmenden Offenheit dem literarischen Erbe gegenüber. In diesem Beitrag wird deshalb auf das intertextuelle Gedächtnis der Texte Monika Marons fokussiert. Ausgehend von ihrem von der Forschung bisher vernachlässigten Stück Ada und Evald und der darin enthaltenen Auseinandersetzung mit den Konzeptualisierungen der literarischen Kommunikation durch Heiner Müller, sollen einzelne Stationen einer Entwicklung, die sich als graduelle Entschränkung der Rhetorik bezeichnen lässt, nachgezeichnet werden.

\section{I.}

Im fünften Bild des wenig gelesenen und bisher nur zweimal aufgeführten Stückes von Monika Maron, Ada und Evald aus dem Jahr 1982, ${ }^{1}$ sitzt eine

\footnotetext{
${ }^{1}$ Monika Maron: Ada und Evald. Ein Stück. In: Das Mißverständnis. Vier Erzählungen und ein Stück. Frankfurt/M. 1982. S. 91-124 (Uraufführung 1983 in Wuppertal, 1986 auch in Zürich). Im Folgenden wird unter der Sigle AE zitiert.
} 
Gestalt namens X, klein, und wie ein Gartenstuhl zusammengeklappt, auf einem Tisch und isst Papier:

Er ißt es gleichmütig, ohne sichtbares Vergnügen, auch ohne Ekel. X sagt: Die griechische und die römische Antike habe ich schon in meinem Bauch, und ich bin immer noch hungrig. Das Zeug sättigt nicht. Aparte Vorspeise zum großen Schlachtefest: Menschen als Löwenfraß, enthäutete Sänger, Mutterschänder. Tragödien tragen Menschennamen: Antigone, Medea, Ödipus; nicht Auschwitz, Stalingrad, Hiroshima. Ich kann Blut nicht sehen. Es würgt mich, und ich muß weinen, wenn ich Blut sehe, und doch treibt mich eine Blutrünstigkeit, die nicht satt werden kann. Ich fresse die blutigen Papierleichen in mich hinein, hundert, zweihundert, und speie sie aus in neuer Gestalt, und schon wieder quält mich der ekle Durst auf neues Blut, als könnte mir nur fremder Tod beweisen, daß ich lebe. Soviel Tod kann es gar nicht geben auf meiner Erde, daß ich einmal tanzen möchte. Einmal soviel Leben fühlen, daß die Beine tanzen müssen. (AE 104)

Die Szene, und eigentlich das gesamte, aus poetischen Bildern aufgebaute - und im Grunde kaum spielbare - Stück, ${ }^{2}$ bringt eine pessimistische Vorstellung von der determinierenden Macht der Geschichte und der Grunderzählungen unserer Zivilisation zum Ausdruck. Der Schriftsteller X hadert in der zitierten Szene mit der Kriegslogik und den vielen anderen, grausamen und lebensverachtenden Relikten aus der griechischen und römischen Antike. In anderen Bildern versucht sich die Titelheldin Ada aus ihrer biblischen Bestimmung als passiver Geliebten des Evald zu befreien. Die von ihr neu erzählte Schöpfungsgeschichte lässt sie mit dem Selbstmord Evas enden. ${ }^{3}$

Das von X praktizierte - in diesem Falle buchstäblich Papier fressende Wiederkäuen der Mythen aus alten Zeiten gleicht der puren Selbstquälerei. In den frühen Werken Monika Marons taucht diese Art des Masochismus aber wiederholt als Merkmal einer typisch "männlichen", zwischen anxiety of influence, Selbsthass und Größenwahnsinn schwankenden Kreativität auf: Fast alle Männergestalten aus Marons Werken der achtziger Jahre leiden unter einem ausgesprochenen Zitationismus. ${ }^{4}$ Evald, der zweite Dichter in Ada und Evald,

${ }^{2}$ Vgl. zu den Schwierigkeiten bei der Wuppertaler Aufführung: Hedda Kage: Wider glatte Vereinnahmung. Rückblick auf die Arbeit an M. Marons Ada und Evald und G. Leuteneggers Lebewohl, Gute Reise. In: TheaterZeitSchrift. Schwerpunkt: Frauen am Theater 9 (1984). S. 6-18.

3 "Wenn ich die Augen schließe, kann ich das Paradies sehen, sagt Ada. Wie sieht es aus, fragt Evald. Eva hängt am Baum der Erkenntnis, die Schlange schläft, Adam pflanzt Vergißmeinnicht. Warum hat sie sich aufgehängt, fragt Evald. Später hätte es sich nicht mehr gelohnt, sagt Ada. Sie hat vom Baum der Erkenntnis gegessen, aber sie hat Adam nichts abgegeben. Und erkannte seine Worte: das ist doch Bein von meinem Bein und Fleisch von meinem Fleisch; man wird sie Männin heißen, darum, daß sie vom Manne genommen ist. Da wollte sie sich den Rest ersparen" (AE 95).

${ }^{4}$ Am auffälligsten betroffen sind der "Graf" Karl Heinz Baron und Bruno aus Die Überläuferin (Frankfurt/M. 1986) und Stille Zeile Sechs (Frankfurt/M. 1991). Vgl. besonders Die Überläuferin. S. 82-86. 
ist ein selbsterklärtes, aber leider völlig talentloses Genie. Für einen Tropfen Nachruhm zerrt er sich die Verse aus dem Leib. Er versteht sich als Nachfolger der großen revolutionären Dichter vergangener Jahrhunderte und will um jeden Preis anerkannt werden als "Bruder [seiner] Brüder Friedrich, Georg und noch einmal Georg" und als "Sohn seiner blutigjungen Stiefväter, die verreckt sind in ihrem blutigjungen Blut, gestorben im Wasser, im Fieber, im Wahnsinn" (AE 97). Aus seiner eingebildeten Zugehörigkeit zu einer ehrenvollen männlichen Traditionslinie speist sich auch Evalds grotesk überzogene und schon dadurch sich selbst entlarvende Misogynie. Angesichts der Unmöglichkeit, es den göttlichen Vorgängern gleichzutun, empfindet er eine Agonie, die er seiner Geliebten nicht erklären möchte: "Auch wenn du dich mühst, begreifst du das nie. Du bist eine Frau und kein Genie" (AE 119).

Evalds Schriftstellerkollege X leidet weniger unter der Größe seiner Vorgänger als unter den Gespenstern, die ihre Geschichten hinterlassen haben. X, der als boshafter Zyniker (und utopiefeindlicher Feigling) dargestellt wird, ${ }^{5}$ wird täglich von den Geistern der Toten heimgesucht. Die blutdürstigen Spukgestalten bewegen ihn dazu, auch seinerseits zu töten, zuerst nur in Gedanken, dann auf Papier: "Ich erfand Menschenfresser, Mörder, Selbstmörder, Töter und Opfer" (AE 106). In seinen Gesprächen mit den Ungeheuern der Vergangenheit nährt sich für $\mathrm{X}$ die Überzeugung, dass das Leben, das alle um ihn herum anbeten, nie etwas anderes hervorgebracht habe als den Tod (AE 105). Aus den Begegnungen mit den Toten hat er darüber hinaus gelernt, dass auch die Zukunft für wirkliche Veränderungen keinen Raum bieten wird. Es besteht keine Chance auf eine Unterbrechung der schicksalhaften Abfolge von Massakern. Für Taten, die dieser und anderen Manifestationsarten der menschlichen Neigung, sich gegenseitig auszumorden, ein Ende bereiten würden, gibt es in der Weltsicht des X keinen Platz. Von dieser hoffnungslosen Einsicht zeugt nicht nur seine wiederholte Bekundung, dass es "keine Schuld" (AE 124) gebe; als Indiz in diese Richtung ist auch sein sarkastischer Bezug auf die Parolen der sozialistischen Arbeiterbewegung zu werten. ${ }^{6}$ Anders als Evald wird der Schriftsteller $\mathrm{X}$ auch in seiner Beurteilung der weiblichen Kreativität eher von einer generellen Misanthropie als von einer spezifischen Misogynie geleitet. Der Zyniker findet "rührend" das "esoterische[...] Gestammel", wenn "die Weiber", wie er sagt, "einfältig wie die Kinder, ihre Geschichte in einer Sprache suchen, in der sie keine haben" (AE 105). Es sei ein großes Glück, keine Geschichte zu haben.

\footnotetext{
${ }^{5}$ Vgl. dazu das Rollenspiel, in dem Clairchen in der Rolle des $\mathrm{X}$ behauptet: “Ob Zukunft oder Himmelreich / die Hoffnungsträume sind alle gleich" (AE 117).

${ }^{6} \mathrm{X}$ begleitet seine Aktionen mit Zeilen aus dem fortschrittsgläubigen und tatendurstigen kommunistischen Kampflied "Brüder seht die rote Fahne weht uns kühn voran" (AE 104) Kaum zu überhören ist der Sarkasmus besonders in dem abschließenden Zitat, in dem auf das erwartete Ende der Qualen Bezug genommen wird: "Wenn die letzte Schlacht geschlagen, Waffen aus der Hand" (AE 107).
} 
Es fällt nicht allzu schwer, in Marons zynischer Schriftstellergestalt eine Karikatur des 1995 verstorbenen Dramatikers Heiner Müller zu erkennen. Dass Ada und Evald außerdem ein paar auffällige Motive aus dessen "Greuelmärchen" Leben Gundlings Friedrich von Preußen Lessings Schlaf Traum Schrei (aus den Jahren 1976/1977) übernimmt, ${ }^{7}$ macht eine Deutung der Maron'schen Figur als Wiedergänger jener bei Müller auftretenden "drei Figurationen eines [einzigen] Traums von Preußen" annehmbar. ${ }^{8}$ Die nach Müller "in vielen Punkten als Selbstporträt" zu betrachtenden Figuren Friedrich, Kleist und Lessing leben in der Schilderung von $\mathrm{X}$ weiter. ${ }^{9}$ Besonders stark sind die Parallelen zu Lessing, der bei Müller "brustkrank" geworden war "vom Staub der Archive und von der Asche, die aus den Büchern weht" und der "gewürgt" wurde von einem "wachsenden Ekel an der Literatur". ${ }^{10}$ Müllers Lessing, der Dichter, dem die Geschichte eine Last ist, der "ein neues Zeitalter nach dem anderen heraufkommen sehn [hat], aus allen Poren Blut Kot Schweiß triefend jedes", sehnt sich nach Vergesslichkeit, denn "Vergessen ist Weisheit". ${ }^{11}$

Die Entsprechungen zu Müllers Dramentext betreffen aber auch andere der Maron'schen Figuren. So, wie seine Preußen alle von einem Doppel in der Gestalt einer Puppe begleitet werden, besitzen auch Marons Protagonisten Ada, Evald und X ein wächsernes Ebenbild, das als Abspaltung ihres früheren Selbst kontinuierlich an die eigene Vorgeschichte erinnert. ${ }^{12}$ In beiden aus fragmentarischen Szenen aufgebauten Stücken übernehmen die Figuren hin und wieder auch die Rollen ihrer Gegenspieler; manchmal werden mehrere Gestalten von einem einzigen Schauspieler dargestellt. Der von Kostümen, Perücken, Masken, Brillen, Hüten und sogar umgeschnallten Lederphalli theatralisch hervorgehobene Rollentausch vermittelt, zusammen mit den Puppen, ein Bild vom Individuum als austauschbarem Subjekt, als fremdgelenkter Marionette, deren Dasein sich in der Wiederholung der ewiggleichen Scripts und Muster erschöpft.

Eine weitere auffällige Gemeinsamkeit zwischen beiden Dramentexten findet sich in der Demontage, die X an seinem Doppel vornimmt. In Müllers Pantomime zerhackt Kleist seine Puppe, um sie danach neu zusammenzusetzen. Auf ähnliche Weise sammelt auch X die verstreut im Raum herumliegenden Teile seiner Puppe ein, um aus ihnen immer neue und vor allem immer hässlichere Konstellationen bilden zu können. Als wäre seine Puppe eine, wie

\footnotetext{
${ }^{7}$ Heiner Müller: Leben Gundlings Friedrich von Preußen Lessings Schlaf Traum Schrei. Ein Greuelmärchen. In: Werke 4. Die Stücke 2. Hg. von Frank Hörnigk. Frankfurt/M. 2001. S. 509-554.

${ }^{8}$ Heiner Müller: Krieg ohne Schlacht. Leben in zwei Diktaturen. Köln 1992. S. 269.

${ }^{9}$ Ebd.

${ }^{10}$ Müller: Leben Gundlings. S. 534.

${ }^{11}$ Ebd.

${ }^{12}$ Vgl. Adas Seufzer: "Ach ja, so war ich, wie werd ich noch werden” (AE 93).
} 


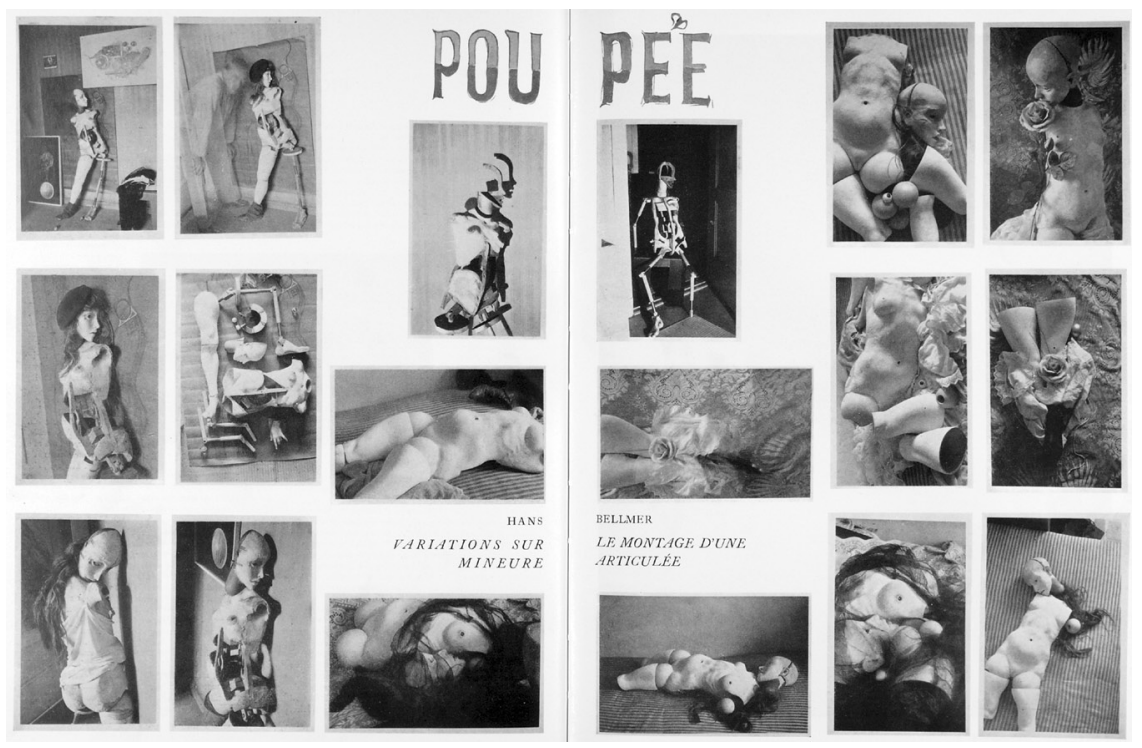

Abb. 2. (C) Hans Bellmer, Poupée, variations sur le montage d'une mineure articulée, Minotaure 6 (Winter, 1934-1935), c/o Beeldrecht Amsterdam 2007.

der Surrealist Hans Bellmer sie in den dreißiger Jahren des vergangenen Jahrhunderts in vielen Varianten produziert hat, schraubt X "den Kopf an die Schultern, einen Arm an die Hüfte, den anderen Arm montiert er anstelle eines Beines. [...] Er schraubt einen Arm an den Hals. [...] verschraubt den Kopf der Puppe in ihrem Hintern" (AE 104-107; Abb. 2). Die Art und Weise, wie X mit seiner Puppe verfährt, wird damit zu einem Sinnbild seiner wiederkäuenden literarischen Produktion: Auch im Umgang mit der Puppe liegt das Neue nur in der veränderten Anordnungsweise, in der, sei es vielleicht unheimlichen und furchterregenden, Reorganisation der existierenden Bruchteile von bereits aus der Vergangenheit Überliefertem.

Die eben durchgeführte Rekonstruktion der Verwendung von Fragmenten aus Müllers Text in Ada und Evald hat ein paar Pfeiler der zentralen These dieses Beitrags schon benannt: Gerade in der Auseinandersetzung mit dem Müller'schen Geschichts- und Literaturverständnis, so die weiter zu entfaltende Annahme, lässt sich der Kern erkennen von einem fortwährenden Versuch der Positionierung gegenüber dem literarischen Erbe, welcher sich durch das ganze Euvre Monika Marons hindurch verfolgen lässt. Die Evolution in den Verhaltensweisen gegenüber den Echos der Toten in Marons Texten - und in einem allgemeineren Sinne auch gegenüber der Prägung durch dasjenige, was vor dem Subjekt bereits dagewesen ist - soll jetzt anhand einzelner Stationen im Werk (und womöglich mit fortgesetztem Bezug auf Müller) nachgezeichnet werden. 
II.

Auch in Die Überläuferin, dem Roman aus dem Jahre 1986, den Maron in Ada und Evald besonders auf der Ebene der Figurenkonstellation schon vorbereitet hatte, tauchen Motive aus Müllers Leben Gundlings auf. Die Historikerin Rosalind Polkowski, Protagonistin in Marons zweitem und drittem Roman, die sich zugunsten eines Lebens im Kopfe aus der Welt der Werktätigen zurückgezogen hat, vollzieht in der Überläuferin einen phantasmagorischen Streifzug durch Berlin und wird dabei Zeuge einer Begegnung zwischen "Heinrich", einem "führenden Mitglied der Assoziation dichtender Männer" und dem eigenen, seit einiger Zeit verschollenen, dichterischen Alter Ego, Martha Mantel. ${ }^{13}$ Heinrich trägt sowohl Züge von Heinrich von Kleist wie von Heinrich Heine und überlagert sich außerdem mit Müllers Friedrich aus Leben Gundlings. ${ }^{14}$ Weil sie die ihr abverlangte "zuverlässige literarische Abstinenz" (Ü 155ff.) nicht eingehalten hat, sieht der Dichter sich genötigt, Martha zu töten. Er erklärt auch, weshalb er der Schriftstellerin das Recht auf weitere kreative Tätigkeit entnehmen soll. Martha solle sich einen Turm vorstellen:

Jede Generation hat ihre Steine in sorgsamer Ordnung, das Werk der Ahnen achtend, hinzugefügt. Goldenes Bauwerk findest du neben Kalkstein, Sandhaufen zwischen Silberklumpen, aber es trägt sich. Das ist die Dichtung, der in Form geronnene Geist der Menschheit, verstehst du. Was glaubst du, würde geschehen, wollte man plötzlich die nächste Schicht bauen zum einen Teil, wie bisher, zum anderen Teil aus Wind, Sonnenstrahlen und Wellenschaum. (Ü 158f.)

Zum prachtvollen Turm der Dichtung, der von den Männern in zweitausend Jahren sorgfältig und Schicht für Schicht errichtet wurde, könne Martha nichts hinzufügen als einen armseligen Haufen Geröll, der unbrauchbarer Baugrund wäre für die Erben. ${ }^{15}$ Da Schreibverbote bisher nicht den gewünschten Effekt erzielt hätten und die anmaßenden weiblichen Verletzungen der gesicherten literarischen Werte sich allmählich von Einzelerscheinungen auf eine richtige

\footnotetext{
${ }^{13}$ Maron: Die Überläuferin. S. 155. Im Folgenden wird unter der Sigle Ü zitiert.

${ }^{14}$ Gemeinsam ist ihnen unter anderem eine Faszination für tote Frauen.

${ }^{15}$ In ihrem Vortrag anlässlich der Eröffnung des Studiengangs Gender-Studies an der Humboldt-Universität Berlin 1997 weist Christina von Braun darauf hin, dass die Verbindung von weiblicher Kreativität mit der Instabilität eines Sandhaufens eine lange Tradition hat (Christina von Braun: Warum Gender-Studies? Vortrag anläßlich der feierlichen Eröffnung des Studiengangs Gender-Studies. 21. Oktober 1997. $<$ http://edoc.hu-berlin.de/humboldt-vl/braun-christina-von/PDF/Braun.pdf $>$ ). Die Denkfigur wurde zum Beispiel auch vom Jura-Professor Otto Gierke benutzt, als er über die Fähigkeit der Frau, an einer Universität zu studieren, zu urteilen hatte. Vgl. dazu Die Akademische Frau. Gutachten hervorragender Universitätsprofessoren, Frauenlehrer und Schriftsteller über die Befähigung der Frau zum wissenschaftlichen Studium und Berufe. Hg. von Arthur Kirchhoff. Berlin 1897. S. $25 \mathrm{f}$.
} 
Volksseuche auszudehnen drohten, will Heinrich das Problem lieber auf seine Art und Weise - mittels Ermordung - lösen. Zuvor klärt er Martha noch über die Grundsätze seiner eigenen Dichtung auf. Heinrich - der sich dadurch auch an HEIN-er Müller selbst anlehnt - führt sich als Verteidiger einer an Artaud erinnernden Ästhetik der Grausamkeit auf: "Die Schönheit ist ein Anachronismus", behauptet er, "man muß sie kaputt machen, damit sie den Blick nicht trübt für das Häßliche und das Böse" (Ü 160).

Anders als in Müllers Idealvorstellung des Theaters, aber, dient das obsessive Interesse des männlichen Dichters für den Gräuel hier anscheinend nicht der Abwehr oder dem Erkennen von Mustern der Ausbeutung und Viktimisierung. Die von Müller anvisierte Wiederbelebung der Untoten auf der Bühne sollte aus dem Theater einen Ort machen, an dem die Begräbnisse der Unbeerdigten der Vergangenheit nachgeholt werden können. In der Heinrich'schen Variante scheint sie jedoch eher zur Gewöhnung und zu einer Einübung der Boshaftigkeit und Lebensfeindlichkeit zu führen, als dass sie in der ständigen Wiederholung zum Aufreißen der fatalen Kette Anlass bieten würde. ${ }^{16}$ Durch ihren Bühnenauftritt wird die Gewaltsamkeit der Ungeheuer eher am Leben gehalten als ausgelöscht. Heinrichs Faszination für Leichen, und besonders für tote Frauen, wird dadurch zu einer selffulfilling prophecy, zu einer fatalen Ausdehnung der grausamen Geschichte, in der Frauen auf ewig keinen Platz finden sollen. Auch wenn die Dichterin Martha ihrer bevorstehenden Ermordung gerade noch zu entkommen weiß, demonstriert doch Rosalinds Alptraum ganz eindeutig eine Angst davor, von den Repräsentanten der (männlich determinierten) Tradition erdrückt zu werden.

Das Gegenbild zu dieser Angstvision bildet eine an anderer Stelle von Rosalind entwickelte Assoziation von Martha mit dem schönen wilden Kind, das auf dem Gemälde La Guerre (1894) von Henri Rousseau zu sehen ist: Das weißgefiederte Reiterkind stürmt auf seinem Pferd über seine "verstümmelten, von Krähen schon zerhackten Opfer" (Ü 138; Abb. 3). Unerschrocken und in unschuldiger Bosheit galoppiert das Kind über die Leichen der Verstorbenen. In den Augen der Surrealisten galt der Maler Henri Rousseau als pures, nicht durch bewusstes Lernen verdorbenes Genie, als einer, dessen Augen viel weiter sehen konnten als die der ausgebildeten Künstler. Dass gerade ein Bild von diesem Künstler der Idealvorstellung von Martha entsprechen sollte, signalisiert, inwiefern ihre poetologische Position als Dichterin ohne literarische Tradition von Rosalind als Triumph über die Toten bewertet wird. Marthas Versuch, unbeeindruckt durch Vorbilder und Regeln, also ex nihilo zu kreieren, führt aber ins Scheitern. Sie endet nicht als erfolgreiche Lyrikerin, sondern

${ }^{16}$ Vgl. dazu unter anderem Müllers Gespräch mit Alexander Kluge: Auf dem Weg zu einem Theater der Finsternisse. In: Alexander Kluge und Heiner Müller: Ich bin ein Landvermesser. Gespräche. Neue Folge. Hamburg 1996. S. 67-88. 


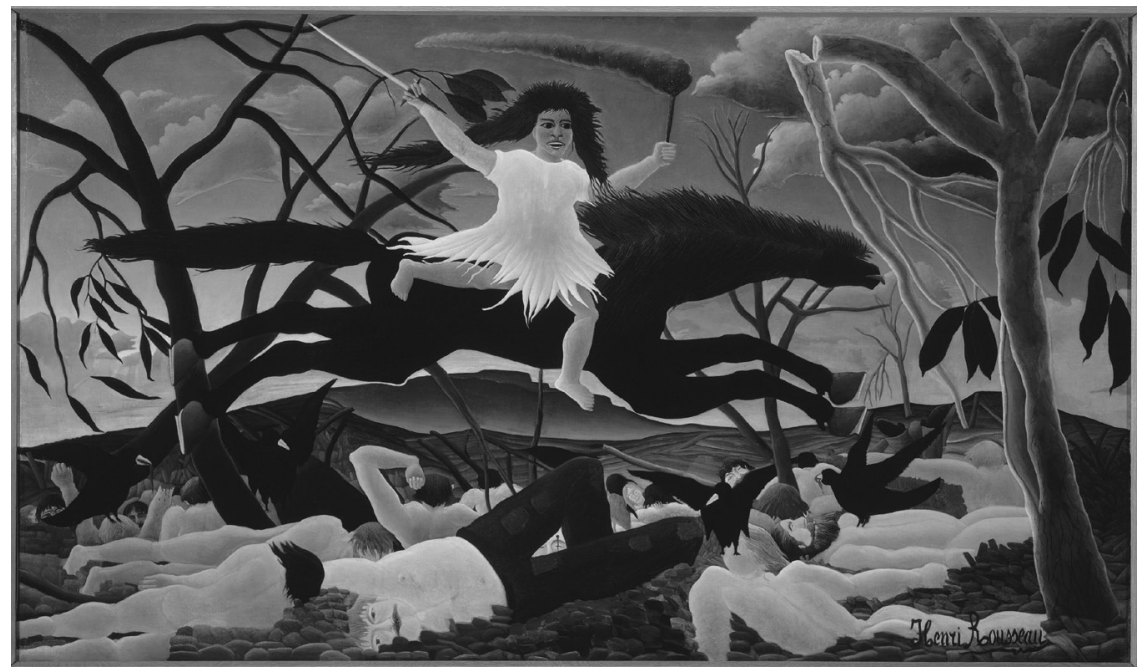

Abb. 3. Henri Rousseau, La Guerre ou la chevauchée de la discorde, Paris, musée d'Orsay, (C) Photo RMN / C Droits réservés.

wird von Rosalind letztendlich in der Gosse im New Yorker Obdachlosenviertel The Bowery aufgefunden.

Mit ihrem Rückzug aus der Welt und aus den Banalitäten des Alltags zugunsten eines Lebens im Kopf versucht die Protagonistin auch selber die Unvoreingenommenheit zu erreichen, welche Marthas Verachtung für den Kanon anfänglich zu verheißen scheint. In vier grotesken "Zwischenspielen" auch dies ein von Müller gern benutztes Gestaltungsprinzip - wird die Heldin aber mit typisierten Vertretern der vorgefassten Meinungen ihres Alltags konfrontiert. Die plötzlich auftauchenden Gespenster der Vergangenheit können als Personifikationen der von Rosalind in Herkunft, Erziehung und Veranlagung empfundenen Bedrängnis gelesen werden. Die Namen, die sie bei Maron erhalten - "die Frau mit der eigenen Meinung", "die Frau mit dem zarten Wesen", "die Frau mit der hohen Stimme", "der Mann mit der traurigen Kindheit" und so weiter - sind in ihrem Aufbau an diejenigen angelehnt, welche in Leben Gundlings zur Bezeichnung der Figuren in einem Bild der Zukunft verwendet werden. Im Traum sieht Müllers Lessing: "Die Frau am Strick Die Frau mit den aufgeschnittenen Pulsadern Die Frau mit der Überdosis [...] Die Frau mit dem Kopf im Gasherd". ${ }^{17}$

Die gespenstischen Wiedergänger ihrer Vergangenheit, von denen sie in den Zwischenspielen heimgesucht wird, möchte Rosalind mit einer damnatio

${ }^{17}$ Müller: Leben Gundlings. S. 533f. 
memoriae belegen: ${ }^{18}$ "Ich werde sie vergessen, ich werde einfach die Falltür in meiner rechten Schläfe öffnen und sie ins Vergessen stürzen" (Ü 180). Doch der Versuch, alles Erlernte durch eine bewusste Entscheidung aus dem Gedächtnis zu tilgen, um völlig neu anfangen zu können, scheitert kläglich: Die Verdammten gehorchen ihrer Entscheidung nicht, sie kommen der Protagonistin auch ungerufen noch ins Zimmer. Die Zwischenspiele versinnbildlichen, inwiefern Rosalind, auch nachdem sie sich von allen ihr denkbaren äußeren Zwängen und sogar körperlichen Bedürfnissen befreit hat, in ihrem Kopftheater weiterhin von den vorgefertigten Meinungen der Geister ihres bisherigen Lebens verfolgt wird. Manche Figur bleibt in ihrem Zimmer sogar als versteinertes Denkmal zurück.

Die damit angesprochene Angst, keinen eigenen Platz zu finden, weil die Toten und Untoten vergangener Zeiten immer noch da sind, treibt auch andere von Marons Protagonistinnen um. Annaeva, die Heldin aus der gleichnamigen Geschichte (1982) ${ }^{19}$ die - wie Martha Mantel - Angst davor hat, ein bloßes "Plagiat" zu sein, ${ }^{20}$ meidet die Bücher, weil sie ihr beweisen, dass sie überflüssig ist:

Nichts das es nicht schon gegeben hätte, kein Schmerz, der nicht längst empfunden wurde, keine Freude, die nicht schon widerlegt war, jede Besonderheit war schon Allgemeingut. [...] In [den Büchern] war ihr Leben längst beschrieben, vollendet, gefeilt und geschliffen; sie mußte es nicht mehr leben. (A 36)

Weil sie "Beweise ihrer lebendigen Existenz" (A 36) finden möchte, welche die vertrauten Muster, die "Riten und Floskeln" (A 38) ihres Alltags widerlegen könnten, verlässt Annaeva eines Tages das Land, in dem sie aufgewachsen ist. Sie zieht in die Dürre und fängt im Gehen schon mit dem Vergessen an: "Ich vergesse meine Mutter, ich vergesse meinen Vater, niemand hat mich geboren, ich bin der Erbe von nichts" (A 35).

Annaevas durchgreifender Versuch, sich vom Gelernten, von den alten Denkweisen sowie von allen bisherigen Weggefährten zu trennen - von den "Freunden, die sie nicht mehr liebte" und den "Feinden, die sie nicht mehr haßte" (A 33) -, wird begleitet von der Entscheidung, neues Vertrauen in das Erkenntnispotential der rein körperlichen Wahrnehmung zu gewinnen: "gedachte Gedanken, gefühlte Gefühle, gelernte Begriffe will ich vergessen und will meinen Ohren trauen, meinen Augen und meiner Haut" (A 37). Die Sinne der Protagonistin aber erinnern sich weiterhin und machen ihr die Unterscheidung

\footnotetext{
${ }^{18}$ Vgl. zur damnatio memoriae auch Harald Weinrich: Lethe. Kunst und Kritik des Vergessens. München 1997. S. 51f.

${ }^{19}$ Monika Maron: Annaeva. In: Das Mißverständnis. Vier Erzählungen und ein Stück. Frankfurt/M. 1982. S. 31-45. Im Folgenden wird unter der Sigle A zitiert.

${ }^{20}$ Vgl. "Keine Fremden mehr, keine Geheimnisse, nur noch Plagiate" (Ü 101, 207).
} 
zwischen Einbildung und realer Empfindung, zwischen Simulation und Authentizität unmöglich. ${ }^{21}$ Es lässt sich nicht entscheiden, ob eklige grüne Spinnen und Säbelzahnameisen wirklich eine klebrige Schleimspur auf Annaevas Arm hinterlassen. Genauso plausibel wäre, dass ihr "deutlich fühlender Körper" (A 38) die sagenumwobenen Tiere nur spürt, weil Annaeva, die mit vielen mythischen Vorstellungen von der Dürre aufgewachsen ist, deren Attacken schon erwartet. Bei der Wahrnehmung ihrer Umgebung begegnet die Heldin dieser Fluchtgeschichte eines Tages sich selbst: In der Wüstenlandschaft trifft sie auf Spuren der eigenen Erinnerung, die sie mit den Stimmen anderer verwechselt:

Woher kamen diese Sprüche. Sie hingen wie fremde Erinnerungen über der Landschaft. Wessen Erinnerungen. Wer war hier schon gegangen. Wo lagen seine sonnengebleichten Knochen. [...] Oder es waren ihre eigenen Gedanken, die, schon zur Erinnerung geronnen, fremd auf sie niederkamen. (A 42)

Annaeva, die nur noch ihren Sinnen trauen möchte, trifft nicht auf das, was in der Außenwelt zu sehen wäre, sondern auf ihre eigenen Gedächtnisbilder. Sie läuft den Gespenstern so lange davon, bis sie schließlich ankommt am "Stadttor von Nirgends", wo "Niemand" (A 44) auf sie wartet.

Die beschriebene Art des Ent- und Rückzugs, auch des verzweifelten, letztlich aber aussichtslosen Kampfes ist für den Umgang mit den Spuren einer als bedrohlich empfundenen individuellen und kollektiven Vergangenheit in Marons Werk aus den achtziger Jahren durchaus typisch. In ihrem Misslingen jedoch, wird die obsessive Sehnsucht der Protagonistinnen nach der totalen Tabula rasa stets $a d$ absurdum geführt. Die zahlreichen Versuche, das Eigene vom Fremden zu trennen - und das Echte von der Simulation -, werden in den Texten häufig auch ironisch kommentiert; entweder, im Rückblick, durch die Protagonistin selber oder durch andere Figuren. Angesichts des von Rosalind in der Überläuferin geäußerten Wunsches, "mit sich eins zu werden", behauptet ihre Freundin Clairchen zum Beispiel, sie hätte sich über diese Art der "Paradiessucht" immer schon "dodlachen können" (Ü 120). Ironische Kommentare begleiten auch die in wirklich jedem Text Marons vorkommenden Träume von der Naturkatastrophe, der Flutwelle oder der Sonnenglut, welche den Neuanfang durch endgültige und restlose Vernichtung des Existierenden ermöglichen sollen: So, wenn Rosalind in der Überläuferin behauptet, sie "hätte gern ein Erdbeben" (Ü 140), wenn sie in Stille Zeile Sechs erwartet, dass sich der "verdammte Himmel" auftun möge und eine "Sturzflut" oder "Sonnenglut" niederlasse, "die Brände entzünden kann", 22

${ }^{21}$ Vgl. zur "erinnernden Wahrnehmung” Peter Matussek: Der selbstbezügliche Blick. Ein Merkmal des erinnernden Sehens und seine medialen Metamorphosen. In: Visualität. Sichtbarkeit und Imagination im Medienwandel. Hg. von Horst Wenzel. Bern 1999. S. 637-654 (Zeitschrift für Germanistik. Neue Folge. IX:3).

${ }^{22}$ Monika Maron: Stille Zeile Sechs. Roman. Frankfurt/M. 1991. S. 216. 
oder wenn der Wanderprediger in Ada und Evald, in einer "Mitteilung der Börse für interessierte Laien", verkündet, dass "wegen der großen Nachfrage" (AE 114) der Wert von Katastrophen weiter ansteige.

III.

Die politische "Wende", die sich im Jahre 1989 vollzog, war ein solches Naturereignis, welches "das Unterste nach oben und das Oberste nach unten" zu wälzen vermochte. ${ }^{23}$ Jedenfalls wird das Ereignis in Marons Texten der neunziger Jahre als eine solche "dramatische Klimaveränderung" dargestellt: Mit der Wende geschah etwas "Gewaltiges", "ein Wunder" (E 40), das, wie es in Marons immer noch bekanntestem Nachwenderoman Animal triste (1996) heißt, "größer war als der Mensch und sein wechselhaftes Streben". ${ }^{24}$ Dass das Jahr 1989 auch in der Rezeption des Werkes der vormals ostdeutschen Autorin gemeinhin als radikaler Einschnitt bewertet werden sollte, nimmt aus diesem Grund nicht wunder. Der Bruch im Euvre ist aber nicht so sehr, wie häufig suggeriert wurde, auf thematischer Ebene feststellbar, ${ }^{25}$ und er manifestiert sich auch nicht darin, dass Marons literarische Produktion erst mit der Wende autoreflexiv geworden wäre - wie es in der bisherigen Forschung ebenfalls nahe gelegt wurde. ${ }^{26}$ Alle Geschichten im Werk entstammen der subjektiven Erinnerung der Erzählerin. Dass erst die Prosatexte der neunziger Jahre aber als "Erinnerungsbücher" wahrgenommen - und als solche von den als "Trilogie" bezeichneten drei Romanen Flugasche, Die Überläuferin und Stille Zeile Sechs abgegrenzt - werden, hängt mit einer auffälligen Evolution in der Akzeptanz von Erinnern (und Vergessen) in Marons Euvre zusammen.

Die Bedingungen für die gemeinte, unbeschwertere Art der Hinwendung zur Vergangenheit wurden, paradoxerweise, erst durch die Erlaubnis, zu vergessen, kreiert. Mit dem Verschwinden des Bedürfnisses nach kritischer Stellungnahme zu dem von der Autorin als diktatorisch eingeschätzten Staat (und seinem verlogenen offiziellen Geschichtsverständnis) ging eine neue Fähigkeit und eine neue Bereitschaft, sich zu erinnern, einher. Zum historischen Ereignis trat eine intensivierte Auseinandersetzung mit der im letzten Jahrzehnt exponentiell angewachsenen Theoriebildung zum Gedächtnis hinzu.

\footnotetext{
${ }^{23}$ Monika Maron: Endmoränen. Roman. Frankfurt/M. 2002. S. 40. Im Folgenden wird unter der Sigle E zitiert.

${ }^{24}$ Monika Maron: Animal triste. Roman. Frankfurt/M. 1996. S. 89.

${ }^{25}$ Diese Einschätzung, der zufolge Maron nach der Wende völlig neue Themen entdeckt hätte, prägt einen Großteil der Reaktionen zu Animal triste.

${ }^{26}$ Suggeriert hat das vor allem Andrea Geier: Paradoxien des Erinnerns. Biografisches Erzählen in Animal triste. In: Monika Maron in Perspective. "Dialogische" Einblicke in zeitgeschichtliche, intertextuelle und rezeptionsbezogene Aspekte ihres Werkes. Hg. von Elke Gilson. Amsterdam - New York 2002. S. 93-122 (German Monitor 55).
} 
Die Unversöhnlichkeit von totaler Vergesslichkeit und konstruktiver Tätigkeit, welche in den frühen Texten einfach durch das Scheitern erkennbar gemacht wurde, wird in den Texten der letzten Jahre zunehmend auch theoretisch fundiert. Die Erkenntnis dieser Unversöhnlichkeit hat dazu geführt, dass an die Stelle des oben skizzierten Kampfes mit den Toten ab 1990 in Marons Euvre eine immer überzeugtere Totenbeschwörung getreten ist. Während in den frühen Prosatexten die Protagonistinnen - auch diejenigen, die hier nicht erwähnt werden konnten - sämtlich am Gefühl litten, die Geschichte als konditionierende Macht mache "eigene" Konstruktionen unmöglich, werden Marons Werke der neunziger Jahre eher von der Einsicht gekennzeichnet, dass überhaupt nur etwas zustande kommt, wenn überlieferte Muster für eigene Zwecke neu zusammengesetzt und kombiniert werden.

Der postmodern-unverkrampfte Umgang mit der Tradition in Marons erstem wirklichen Nachwenderoman Animal triste (1996) liest sich als Illustration der von Umberto Eco in seiner Nachschrift zum Namen der Rose formulierten Erkenntnis, dass die Vergangenheit, nachdem sie nun mal nicht zerstört werden kann, da ihre Zerstörung zum Schweigen führt, auf neue Weise ins Auge gefasst werden muss, "mit Ironie, ohne Unschuld" ${ }^{27}$ Wurde X wegen seines Zusammensetzens überlieferter Bruchteile in Ada und Evald noch der Lächerlichkeit ausgesetzt, so spielt jetzt der Liebesroman selbst mit einer Vielzahl von existierenden, literarischen Modellen, welche die Musterhaftigkeit der in ihm dargestellten Liebeserfahrung explizit zur Schau stellen: "Tristan und Isolde, Romeo und Julia, Anna Karenina, Paolo und Francesca, Penthesilea", 28 "Ferdinand und Luise, Philemon und Baucis", 29 "Orpheus und Euridyke", ${ }^{30}$ alle Liebesgeschichten vergangener Jahrhunderte werden von der Erzählerin mit der ihrigen amalgamiert. Die Unfähigkeit, zwischen dem wirklich Geschehenen und dem Erfundenen (oder dem wegen der Modelle Erwartbaren) zu unterscheiden, wird in der Erzählstruktur von Animal triste zum strategischen Prinzip, das der Irreführung des Lesers dient. ${ }^{31}$

Das Hadern mit der unausrottbaren Wirkungsmacht von Schemata, "Scripts" und "Frames", durch die eigene Denk-, Wahrnehmungs- und Handlungsweisen

\footnotetext{
${ }^{27}$ Umberto Eco: Nachschrift zum "Namen der Rose”. München 1984. S. 79.

${ }^{28}$ Maron: Animal triste. S. 59.

${ }^{29}$ Ebd. S. 92.

${ }^{30}$ Ebd. S. 58.

${ }^{31}$ Relevant wird die Unfähigkeit, zwischen dem Faktischen und dem Imaginierten zu unterscheiden, besonders in Bezug auf die Mordgeschichte im Roman: Da die Protagonistin sich im ganzen Roman auffällig stark mit Penthesilea identifiziert, bleibt bis zuletzt unklar, ob die am Ende angeblich "erinnerte" Ermordung des Geliebten tatsächlich stattgefunden hat oder nicht. Vgl. dazu ausführlicher Henk Harbers: Gefährliche Freiheit. $\mathrm{Zu}$ einem Motivkomplex im Werk von Monika Maron. In: Monika Maron in Perspective. S. 123-137.
} 
gelenkt werden, ist damit in Animal triste einer mehr oder weniger bewussten Aneignung gewichen. In diesem "vielstimmigen Monolog"-wie der Rückblick der Ich-Erzählerin in einer Rezension bezeichnet wurde - wird das, was früher noch als "aufoktroyiert" und "fremd" zurückgewiesen wurde, als zum "Eigenen" gehörend anerkannt. ${ }^{32}$ Mit seiner intertextuellen Akzentuierung der Tatsache, dass jedes verwendete Wort schon von den Worten anderer überlagert ist, bestätigt der in Erzählhaltung und -situation an Beckett erinnernde und eigentlich solipsismusverdächtige Roman auf eindrucksvolle Weise Bakhtins Befund über den "illusorischen Charakter der Einsamkeit". ${ }^{33}$

Karl Heinz Baron, die liebenswürdigste Gestalt in Marons zweitem Roman Die Überläuferin, Sinologe, der sich auch durch Dutzende von anderen Sprachen bereits vollständig "hindurchgedacht" (Ü 82) hat, sehnt sich immer wieder nach einer weiteren neuen Sprache, weil jede neue Sprache "wie ein neues Leben sei" (Ü 84), in dem man die eigenen Gedanken zum ersten Mal denken könne. Im zehn Jahre später erschienenen Animal triste dagegen wird die dialogische, palimpsestartige Struktur eines jeden Wortes selbstbewusst und spielerisch erkundet: Nicht der eine, authentische, höchst individuelle und nur für dieses sprechende Subjekt gültige Ausdruck, sondern die immer sich verschiebenden, nie bewusst oder endgültig unter Kontrolle zu bringenden Bedeutungsebenen des Wortes werden in diesem Text ausgeschöpft. "Schlimm, schlimm, schlimm," nannte der Graf das unheimliche "Geheimnis der Reminiszenzen" (Ü 166), das darin bestünde, dass keine Wege noch allein zu begehen wären. Die - im oben Zitierten - auch von Annaeva gemachte Erfahrung, dass kede Wahrnehmung zur Selbstbegegnung führt, und in der Erinnerung an die eigenen Prägungen auch zur Begegnung mit den Stimmen der Anderen, war dem Grafen noch unerträglich. In Marons späteren Werken wird jenes "Geheimnis" auf immer neue Art und Weise, "mit Ironie und ohne Unschuld", inszeniert.

Dass der Graf anlässlich seiner Wahrnehmung der Landschaft auf das "Geheimnis der Reminiszenzen" zu sprechen kommt, deutet zugleich auf seine Anerkennung der grundsätzlichen Konstruktivität aller Wahrnehmung, die, in diesem Sinne als Begegnung oder Selbstbegegnung betrachtet, mit der Perzeption als Abbildung des in der Außenwelt "wirklich" Gegebenen wenig zu tun hat. Die Wahrnehmung wird damit auch zur zweifelhaften Grundlage für die Erinnerung an das tatsächlich Gewesene. Das Erinnern, das durch nachträgliches Ordnen, Feilen und Schleifen die Vergangenheit narrativisiert, wurde in Ada und Evald von $\mathrm{X}$ schon zynisch kommentiert. Die beim

\footnotetext{
${ }^{32}$ Vgl. Ruth Schweikert: Die Liebe ist ein gar trauriges Tier. Leben, vom Mauerbau bestimmt: Animal Triste, Monika Marons vielstimmiger Monolog. In: Die Weltwoche 22.02.1996.

${ }^{33}$ Diesen "illusory character of solitude" erläutert Mikhail Bakhtin in: Toward a Reworking of the Dostoevsky Book. In: Problems of Dostoevsky's Poetics. Hg. von Caryl Emerson. Minneapolis 1984. S. 283-302.
} 
Vergegenwärtigen der Vergangenheit fast unumgängliche Legendenbildung hatte X als Ausstattung der Geschichte mit "Schleifchen" und "Fältchen" beschrieben, als Prozess, bei dem alle Ecken und Risse des Gesteins der Vergangenheit überdeckt und geglättet werden (AE 106). Ein ähnliches Bild verwendet die Erzählerin von Animal triste zur (selbstentlarvenden) Veranschaulichung auch ihrer eigenen rekonstruktiven Erinnerungsarbeit. Erinnerungen kämen sowieso zustande, sagt die im ganzen Roman namenlos bleibende Protagonistin, wie die Perlen im Innern der Muschelschale: sie sind

zuerst nur ein lästiger Eindringling ins Muschelfleisch, den die Muschel mit ihrem Mantelepithel umschließt und eine Perlmuttschicht nach der anderen um ihn wachsen läßt, bis ein schillerndes, rundes Gebilde mit glatter Oberfläche entsteht. ${ }^{34}$

Als eine solche nachträgliche Konstruktion, als jeweils den aktuellen Umständen und Bedürfnissen angepasste "Neuinszenierung" vergangener Erlebnisse ${ }^{35}$ welche die Unwiederbringlichkeit der Vergangenheit, "so wie sie gewesen ist", ganz explizit reflektiert, wird die Erinnerung auch in Marons Familiengeschichte Pawels Briefe aus dem Jahre 1999 dargestellt. Das nichtfiktionale Werk, in dem die Autorin anhand der Briefe ihres Großvaters Pawels und der Notizen ihrer Mutter sowie anhand von Fotos und sonstigen Dokumenten die Geschichte ihrer Eltern und Großeltern erzählt, verarbeitet Konzepte sowohl aus der Systemtheorie und der Kybernetik zweiter Ordnung, wie aus neueren hirnwissenschaftlichen Untersuchungen zum Funktionieren des Gedächtnisses. Auf dieser Grundlage entfaltet sich in Pawels Briefe eine konstruktivistische Vorstellung vom Gedächtnis nicht als (räumlich lokalisierbarem) "Archiv", als "Magazin" oder "Speicher", sondern als Funktion, welche alle aktuellen Operationen von "erinnernden Systemen" begleitet. ${ }^{36}$

Einen vorläufigen Höhepunkt erreicht die Tendenz zur selbstbewussten Positionierung gegenüber dem Erbe und den Spuren der Vergangenheit in Marons Roman Endmoränen aus dem Jahre 2002. Die Metapher der alles vernichtenden

\footnotetext{
${ }^{34}$ Maron: Animal triste. S. 106.

${ }^{35}$ Monika Maron: Pawels Briefe. Eine Familiengeschichte. Frankfurt/M. 1999. S. 167.

${ }^{36}$ Von der Vorstellung des Gedächtnisses als Speicher distanziert sich die Autorin auch durch die Kontrastierung ihres eigenen Erinnerungskonzepts mit dem ihrer Mutter. Hella Maron könne, "befragt nach bestimmten Ereignissen aus ihrem Leben, in einem Regal mit der Jahreszahl 1932 oder 1945 oder 1976 nach diesem bestimmten Päckchen" suchen und so "ein naturbelassenes Stück Erinnerung voller Düfte, Temperaturen und Geräusche" finden, das so anmute, "als wäre es gerade erst verpackt [...] worden" (Maron: Pawels Briefe. S. 166f.) Vgl. zu Marons an Luhmann angelehnter Erinnerungsvorstellung Elke Gilson: "Nur wenige kurze Augenblicke, die sicher sind". Zur konstruktivistisch inspirierten Darstellung des Erinnerns und Vergessens in Monika Marons Familiengeschichte Pawels Briefe. In: Colloquia Germanica 33.3 (2000). S. $275-288$.
} 
Naturkatastrophe ist für die Geschichtsvorstellung auch in diesem Roman konstitutiv, nur erhält sie hier eine völlig neue Dimension: Die Endmoränen des Titels verweisen nämlich nicht nur auf die vorpommersche Landschaft, vor der sich das Geschilderte abspielt; mit ihnen werden zugleich auch jene Relikte angesprochen, welche nach einem Erdrutsch noch zurückbleiben. Die Endmoränen deuten auf den Schutt, der - nicht zuletzt im Sehen und Denken - von der Eiszeit hinterlassen wurde; sie symbolisieren das Geröll, das auch nach der drastischen Klimaveränderung noch an die frostige Vorzeit erinnert. Unter den damit vom Titel schon metaphorisch festgehaltenen Abhängigkeiten wird in Endmoränen in der Regel nicht mehr gelitten; in den meisten Fällen werden sie sogar umarmt: Durch zahlreiche explizite intertextuelle Verweise werden die Toten in diesem Roman zum Sprechen eingeladen. Waren die surrealistischen Anklänge (zum Beispiel an Bretons Nadja und an seine Manifeste) in der Überläuferin noch sehr impliziter Natur, so werden in Marons jüngstem Roman die Gewährsmänner (und -frauen) auch namentlich aufgeführt: Neben Max Ernst, Leonora Carrington und Alfred Kubin sind in Endmoränen auch Koestler, Döblin, Camus, Arendt und Solschenizyn vertreten (E 50, 52-57, 152). ${ }^{37}$

Einleuchtend für die Erläuterung des gewandelten Verhältnisses zu den Prägungen des eigenen Denkens ist der Gebrauch, der in Endmoränen von Christa Wolfs Erfolgserzählung Der Geteilte Himmel aus dem Jahre 1963 gemacht wird. ${ }^{38}$ Marons Protagonistin Johanna erinnert sich daran, sich vor vielen Jahren mit ihrer Freundin Elli über das Buch gestritten zu haben: Während sie von sich selbst vermutet, dass sie den Roman "damals wohl gemocht" haben muss, wird von Elli die trockene Mitteilung überliefert, sie lehne es ab, ein Buch zu lesen, "in dem der Satz vorkommt: 'Die Klinke war so eiskalt wie ein ganzes einsames Leben' " (E 118f.). Es fällt schwer, in diesem Urteil über die Kitschverdächtigkeit von Wolfs Geteiltem Himmel nicht eine Stellungnahme und vielleicht sogar eine nachträgliche Desavouierung der ursprünglichen wenigstens motivischen - Nähe von Marons ersten Romanen zu Christa Wolfs Prosa der sechziger und siebziger Jahre zu erkennen. ${ }^{39}$ Die unüberhörbare Herabschätzung wird jedoch schon dadurch gebrochen, dass die geringschätzige Aussage nicht der Protagonistin, sondern ihrer Freundin in den Mund gelegt wird. Später im Roman erfolgt eine zweite Brechung, als die Erzählerin versucht,

\footnotetext{
${ }^{37} \mathrm{Vgl}$. zu den surrealistischen Intertexten das betreffende Kapitel in meiner Dissertation: Ich sehe was, was du nicht siehst. Strategien des Weltenbaus und intertextuelle Muster im Euvre von Monika Maron. Gent 2004. S. 230-256.

${ }^{38}$ Christa Wolf: Der geteilte Himmel. Erzählung. Halle 1963.

${ }^{39}$ Ziemlich deutliche Korrespondenzen gibt es zum Beispiel zwischen Wolfs Der geteilte Himmel und ihrem späteren Roman Nachdenken über Christa T. (Halle 1968) einerseits, und Marons Flugasche (Frankfurt/M. 1981) und Die Überläuferin andererseits. Vgl. dazu ausführlicher Brigitte Rossbacher: Illusions of Progress. Christa Wolf and the Critique of Science in GDR Women's Literature. New York - Washington 2000.
} 
den von Elli verachteten, schnulzigen Satz im Geteilten Himmel wiederzufinden, was ihr aber nicht gelingt (E 152)..$^{40}$

Gerade das Hinnehmen dieses Nichtgelingens könnte auf eine allmählich größer werdende Gelassenheit deuten, welche Johanna vom verbissenen Suchen nach weiteren "Minderwertigkeitsbeweisen" in Wolfs Geschichte abhält. Die Versöhnlichkeit geht sogar noch weiter: In der Zeit vor dem "Wunder", also vor der politischen Umwälzung, hat Johanna selber ein ganzes Leben lang Biographien von historischen Figuren verfasst, um in ihnen leicht subversive Botschaften zu verstecken. Diese "Klopfzeichen aus dem Untergrund", die vom Zensor jedoch leicht zu übersehen waren, haben nach der Wende ihren Sinn völlig verloren. Obwohl Johanna sich über ihre neue Freiheit eigentlich nur freuen will, muss sie doch zugeben, dass sie die alte Aufregung beim Verstecken einer verbotenen Aussage vermisst. Marons Protagonistin verzweifelt zwar einerseits über ihre Unfähigkeit, das eigene Leben nach dem "Wunder" neu zu erfinden; andererseits wird im Eingeständnis ihrer Ratlosigkeit zugleich eine große Nachsicht erkennbar für das Werk von Autorinnen, die sich, solange die DDR bestand, mit dem Rausch der subversiven Tätigkeit über eine im Grunde nicht hinnehmbare Meinungsgängelei hinweggetröstet haben.

Diese Episode und die expliziteren intertextuellen Bezugnahmen offenbaren, dass Johanna die Stimmen ihrer Vorgänger nicht um jeden Preis aus ihrem Leben zu verbannen versucht. In einem Brief an ihren Freund Christian bekennt sie sich sogar zu einer regelmäßigen "Zwiesprache mit Geistern" (E 208). Bei diesem Dialog mit den Verstorbenen würden die Angesprochenen zwar nicht immer antworten, Johanna könne deren Anwesenheit aber fühlen, wenn sie ganz still sei. In solchen reflexiven Momenten spüre sie deren Luftströme im Zimmer und kämen ihr plötzlich Sätze in den Kopf, die vorher noch nicht dagewesen wären (E 216).

Dass Marons literarische Konstruktionen in den neunziger Jahren verstärkt den Dialog aufsuchen, manifestiert sich nicht nur in den Intertexten, sondern auch auf struktureller Ebene: Mit immer radikaleren Methoden wird in die Texte ein multiperspektivisches Erzählen eingeführt, das Raum bietet für viele, gleichberechtigt nebeneinander auftretende Stimmen. Der auffälligste Beleg für diese Entwicklung bilden die Briefe, welche in Pawels Briefe, aber danach auch in Endmoränen die Stimmen anderer ohne modifizierende Eingriffe durch die Erzählerin zu Worte kommen lassen: In Pawels Briefe wird direkt aus den Briefen Pawels und den Notizen Hellas zitiert; ${ }^{41}$ in Endmoränen

\footnotetext{
${ }^{40}$ Der bei Maron nur sinngemäß zitierte Satz findet sich ganz am Anfang von Wolfs Erzählung: "[...] schon legte sie die Hand auf die Klinke (die war eiskalt und fühllos wie ein ganzes einsames Leben) [...]"(Wolf: Der geteilte Himmel. S. 10).

${ }^{41} \mathrm{Vgl}$. zum polylogischen Aufbau des Werkes Elke Gilson: Vermoedens omtrent Pawel. Over polylogische waarheid bij Monika Maron en Uwe Johnson. In: Jaarboek voor Esthetica 2001. Hg. von Frans van Peperstraten. Tilburg 2001. S. 41-62.
} 
spricht nicht nur Christian in seinen zitierten Briefen mit eigener Stimme, auch Wilhelmine Enke, das Objekt von Johannas neuester Biographie, ist durch ausführliche Übernahmen aus ihrer "Apologie" selber vertreten. ${ }^{42}$ In Bezug auf Marons jüngsten Roman ließe sich sogar behaupten, dass auch die langwierigen Plauderszenen, ${ }^{43}$ in denen endlos über die Folgen des wissenschaftlichen Fortschritts diskutiert wird, der Durchsetzung der Polylogie dienen. Fast unmittelbar, also mit zeitlich kaum spürbarer Verspätung, gibt die Erzählerin in diesen Passagen die Gesprächsaussagen ihrer im Sommerhaus versammelten Freunde wieder. Wegen der geringen zeitlichen Distanz werden die einzelnen Beiträge nicht neu angeordnet; sie werden auf fast ungefilterte Art und Weise reproduziert. ${ }^{44}$

IV.

Die wachsende Großzügigkeit und Unbefangenheit den Stimmen der Vorgänger gegenüber, welche sich in Marons literarischen Texten seit der Wende feststellen lässt, scheint eine Entwicklung nachzuvollziehen, die John Bender und David E. Wellbery in einem kontrovers diskutierten Aufsatz aus dem Jahre 1990 als Tendenz zu einer zunehmenden "Entschränkung der Rhetorik" im Zeitalter der Moderne beschrieben haben. ${ }^{45}$ Nachdem die Rhetorik in der Romantik aus dem Bereich der Ästhetik grundsätzlich verbannt wurde, weil das Schaffen sich nicht länger aus kulturellen Codes und Regeln, sondern nur noch aus der expressiven Subjektivität des absolut unvergleichbaren Genies herleiten wollte, habe die Rhetorik in der Moderne eine Wiedergeburt, allerdings eine in neuer Form, erfahren. Von einer in Lehrbüchern beschriebenen, erlernbaren und strategisch einsetzbaren Technik habe sich die gemeinte moderne Rhetorik in eine fundamentale Bedingung menschlicher Erfahrung und Kommunikation verwandelt. Die Rhetorik, die in der Nachfolge von Nietzsches Generalisierung des Begriffs als Conditio, als Grundbedingung der Existenz aufgefasst - und

\footnotetext{
${ }^{42}$ Wilhelmine von Lichtenau: Apologie der Gräfin Lichtenau gegen die Beschuldigungen mehrerer Schriftsteller. Von ihr selbst entworfen: Nebst einer Auswahl von Briefen an sie. Leipzig 1808.

${ }^{43}$ In seiner Rezension des Romans bezeichnet Clemens Ruthner sie als "Quatschpassagen" (Clemens Ruthner: Nachsommer in preußischer Pampa. Monika Maron schürft im ostdeutschen Lebensraum. In: Der Standard 21.09.2002.)

${ }^{44}$ Gerade die Tatsache, dass nicht gekürzt wurde, ist tatsächlich für eine gewisse Langwierigkeit verantwortlich. Auch diese ließe sich jedoch funktional aufwerten: Die Dialogszenen versinnbildlichen dadurch den "splendiden Ennui", die Verzweiflung der Protagonistin über ihre vom Stillstand gekennzeichnete Lebenssituation; sie untermalen ihr Gefühl, der Welt nichts mehr hinzufügen zu können, ja sogar ganz aus ihr gefallen zu sein: "Lass sie doch ziehen, die Welt" (E 95-137).

${ }^{45}$ John Bender und David E. Wellbery: Die Entschränkung der Rhetorik. In: Texte und Lektüren. Perspektiven in der Literaturwissenschaft. Hg. von Aleida Assmann. Frankfurt/M. 1996. S. 79-104.
} 
deshalb von Bender und Wellbery auch lieber als "Rhetorizität" bezeichnet wird, trägt all jenen unpersönlichen Mustern Rechnung, die in jeder menschlichen Tätigkeit am Werk sind und die im Laufe des vergangenen Jahrhunderts von Psychoanalytikern, Soziologen und Linguisten entdeckt wurden. In der Anerkennung der zivilisatorischen, geschichtlichen, wirtschaftlichen, diskursiven und sprachlichen Geprägtheit, und in der erinnernden Reflexion über die Modelle und Scripts, welche alles individuelle Handeln wie Geisterstimmen begleiten, findet sich für das früher verzweifelt kämpfende und einsame Genie zugleich - auch das ist aus der Analyse von Marons Texten hervorgegangen eine neue Gelegenheit zur Begegnung mit dem Anderen des Subjekts.

Die kleine alternative Geschichte über die Hinwendung zur Erinnerung in Marons Nach-Wende-Texten, die dieser Beitrag zu rekonstruieren versucht hat, wäre damit an ihr vorläufiges Ende gelangt. ${ }^{46}$ Die Darstellung verlangt aber noch einen kurzen Nachtrag über das Vergessen: Das anscheinend Paradoxe an Marons wachsender Aufmerksamkeit für die Bedeutung der Erinnerung besteht darin, dass sie, besonders in Animal triste und Pawels Briefe, von einer entschiedenen Verteidigung des Vergessens begleitet wird. Im Bild von der Erinnerung als Perle, das Maron sich aus Brechts Leben des Galilei geliehen hatte, kommt nicht nur die grundsätzliche Konstruktivität der Erinnerung zum Ausdruck, sondern auch ihre potentielle Schädlichkeit. Für die Auster, nämlich, ist die Perle eigentlich eine Krankheit, die erst von den Menschen zur Kostbarkeit erhoben wurde:

Und kommen Sie mir nicht mit der Schönheit von Phänomenen, die das Alter vergoldet hat! Wissen Sie, wie die Auster Margaritifera ihre Perle produziert? Indem sie in lebensgefährlicher Krankheit einen unerträglichen Fremdkörper, zum Beispiel einen Sandkorn, in eine Schleimkugel einschließt. Sie geht nahezu drauf bei dem Prozeß. Zum Teufel mit der Perle, ich ziehe die gesunde Auster vor. ${ }^{47}$

Damit rückt, neben dem "Nutzen", doch noch einmal der "Nachteil der Historie für das Leben" in den Blick. ${ }^{48}$ Das Verständnis der Rhetorizität als

\footnotetext{
${ }^{46} \mathrm{Vgl}$. für andere, eher thematisch vorgehende Analysen Katharina Bolls Studie Erinnerung und Reflexion. Retrospektive Lebenskonstruktionen im Prosawerk Monika Marons. Würzburg 2002, sowie viele Aufsätze, darunter besonders Lothar Bluhm: "Irgendwann denken wir, muß ich das genau wissen". Der Erinnerungsdiskurs bei Monika Maron. In: Mentalitätswandel in der deutschen Literatur zur Einheit (1990-2000). Hg. von Volker Wehdeking. Berlin 2000. S. 141-151; und Eva Bauer-Lucca: "In den Kellern unserer Gedächtnisse". Erinnerungsmuster und literarische "Vergangenheitsbewältigung" in der Zweiten und Dritten Generation. In: La prosa della riunificazione. Il romanzo in lingua tedesca dopo il 1989. Hg. von Anna Chiarloni. Alessandria 2002. S. 41-69.

${ }^{47}$ Bertolt Brecht: Leben des Galilei. In: Ausgewählte Werke in sechs Bänden. Jubiläumsausgabe zum 100. Geburtstag. Bd. 2. Stücke 2. Frankfurt/M. 1997. S. 65.

${ }^{48}$ Vgl. Friedrich Nietzsche: Vom Nutzen und Nachteil der Historie für das Leben. In: Sämtliche Werke. Kritische Studienausgabe in 15 Bänden. Bd. 1. Hg. von Giorgio Colli und Mazzino Montinari. München [u.a] 1980.
} 
unkontrollierbares Apriori, das bis in die tiefsten Schichten der menschlichen Erfahrung vordringt, hinterlässt zugleich immer noch die Frage nach den verbleibenden Freiräumen für Aktionen. Jede neue Konstruktion muss unter den Bedingungen der verallgemeinerten Rhetorizität notwendigerweise $\mathrm{zu}$ einer trotzigen Widerlegung des längst schon Gewussten werden. Dass zu allem Handeln deswegen Vergessen gehört, wusste natürlich schon Nietzsche. In der zweiten seiner Unzeitgemäßen Betrachtungen hielt er fest, inwiefern besonders für das Anfangen eine "Hülle des Unhistorischen" unverzichtbar ist; ohne sie würde der Mensch "nie angefangen haben und anzufangen wagen". ${ }^{49}$

Diese "Hülle des Unhistorischen" nimmt auch die Erzählerin von Pawels Briefe in Anspruch, wenn sie, nach der Feststellung, dass über die Geschichte ihrer von den Nazis ermordeten Großeltern wenig "Neues" zu sagen ist, weil Zeit- und Leidensgenossen vor ihr schon berichtet haben, trotzdem dazu übergeht, den bestehenden Versionen ihre eigene noch hinzuzufügen. ${ }^{50}$ Ihre Entscheidung kontrastiert stark mit den Flucht- und Ausweichstrategien, die Annaeva und die anderen Heldinnen von Marons frühen Geschichten angesichts der Erkenntnis, dass alles schon einmal beschrieben wurde, entwickelt hatten. Das Vergessen, das dieses "Trotzdem-Konstruieren" verlangt, oder das "Konstruieren mit demütigem Trotz", wie man es auch nennen könnte, ist nicht der voluntaristische Akt, der mit einer bewussten Entscheidung zwischen Erinnern und Vergessen unterscheidet. Eben weil sich das Vergessen dem willentlichen Zugriff entzieht, wird die eingangs von der Protagonistin in Animal triste aufgestellte Behauptung, sie habe "gelernt, [sich] an das, was [sie] vergessen will, nicht zu erinnern", von der Romankonstruktion selber widerlegt. ${ }^{51}$ Das erforderliche Vergessen ist ferner auch nicht die Bewegung, in der die Vergangenheit samt ihren Prägungen restlos vernichtet würde. Es ist schon der Moment, in dem die Bedingungen der eigenen Entscheidungen für einen kurzen Moment ausgeblendet werden. In der Terminologie der Luhmann'schen Systemtheorie hieße das, dass von der in der Moderne unumgänglich gewordenen

${ }^{49}$ Nietzsche: Vom Nutzen und Nachteil. S. 252f. In einem Aufsatz zum Historikertag 2002, in dem sie über die subjektive Handlungsfähigkeit und persönliche Verantwortung des Menschen nachdenkt, spielt Maron selbst auf den Titel von Nietzsches Unzeitgemäßer Betrachtung an: Lebensentwürfe, Zeitenbrüche. Vom Nutzen und Nachteil dunkler Brillen: Wer es sich zu einfach macht beim Rückblick auf seine Geschichte, beraubt sich seiner Biografie. In: Süddeutsche Zeitung 13.09.2002. (Später auch in: Traditionen - Visionen. 44. Deutscher Historikertag in Halle an der Saale vom 10. bis 13. September 2002. Berichtsband. Hg. von Andreas Ranft und Markus Meumann. München - Oldenbourg 2003. S. 245-249.)

${ }^{50}$ Maron: Pawels Briefe. S. 7.

${ }^{51}$ Maron: Animal triste. S. 16. Vgl. zur Widerlegung dieser Behauptung und zur Rückkehr des "Verdrängten": Alison Lewis: Re-Membering the Barbarian: Memory and Repression in Monika Maron's Animal Triste. In: The German Quarterly 71.1 (1998). S. 30-46. 
Beobachtung zweiter Ordnung, oder der Beobachtung der Bedingungen der Beobachtung, abgelassen wird, zugunsten der Handlung oder Konstruktion, die immer eine selbstvergessliche Beobachtung erster Ordnung sein muss. ${ }^{52}$

Ein Vergessen, das in diesem Sinne als Akzeptanz der Blindheiten verstanden wird, ohne die keine Beobachtung zustande kommen kann, könnte damit zu einem "Privileg" werden, nicht nur "der Toten", sondern auch der Lebenden. ${ }^{53}$ Da Selektion die Voraussetzung der Erinnerung überhaupt ist, könnte diese Art des Vergessens möglicherweise auch der Resignation, dem Zynismus und Nihilismus des Müller'schen Geschichtsverständnisses entgegenarbeiten. Das Vergessen dürfte dazu aber nicht mehr als "schuldbeladenes" Verdrängen oder als unzulässiges "Verschweigen" der Toten betrachtet werden: Es entspräche eher einer Attitüde, welche die Gespenster der Vergangenheit nicht mehr aus Angst vor ihrer Übermacht besiegen müsste, und welche sie auch nicht mehr zu immer neuen und immer hässlicheren Konstellationen verstümmeln müsste. Marons Prosatexte aus der Nachwendezeit legen auf jeden Fall nahe, dass sich nur mittels eines solchen demütig-trotzigen Vergessens eine ernsthafte Zwie/sprache mit den Geistern gestalten ließe.

\footnotetext{
${ }^{52} \mathrm{Vgl}$. zu dieser Denkfigur unter anderem Niklas Luhmann: Die Kunst der Gesellschaft. Frankfurt/M. 1995. S. 103: "Man würde nie anfangen können, wenn man alle Möglichkeiten des Anfangens gegeneinander abwägen müßte".

53 "Das Vergessen ist ein Privileg der Toten" (Heiner Müller: Mommsens Block. In: Werke 1. Die Gedichte. Hg. von Frank Hörnigk. Frankfurt/M. 1998. S. 257-263. Hier: S. 260).
} 